\title{
Myo-Pericarditis and Congestive Heart Failure Secondary to Mesalamine Use Associated With Completely Reversible Cardiac Dysfunction
}

\author{
Luke Simon Olivera Yuhico ${ }^{\text {a, }}$, Pankaj Bharati ${ }^{\text {a }}$, Stephen Knohl ${ }^{\text {a }}$
}

\begin{abstract}
Mesalamine is a 5-ASA derivative commonly used to treat ulcerative colitis and mild to moderate crohn's disease. Rarely, it has been associated with myocarditis or pericarditis. Several hypotheses have been proposed but the exact mechanism of cardiac injury is still unclear. We present a case of combined pericarditis and myocarditis in a young man on mesalamine therapy, with subsequent deterioration of cardiac function, EKG changes and signs of congestive heart failure. After the appropriate treatment, beginning with discontinuation of mesalamine, he had complete recovery of cardiac function to normal. This case exemplifies the importance of quick physician recognition of the disease association and immediate discontinuation of the drug for more successful outcomes in conjunction with appropriate management.
\end{abstract}

Keywords: Mesalamine; Congestive heart failure; Myopericarditis; Drug effect

\section{Introduction}

Mesalamine, a 5-ASA derivative, is typically known for its use in the management of inflammatory bowel disease. Although it is more commonly associated with adverse reactions such as headache, gastrointestinal discomfort, hypersensitivity and hepatic/renal toxicity, it has been described to cause both pericarditis and myocarditis. Physician awareness of this association is crucial as immediate discontinuation of

\footnotetext{
Manuscript accepted for publication March 13, 2012

${ }^{a}$ Department Of Medicine, SUNY Upstate Medical University, USA ${ }^{\mathrm{b}}$ Corresponding author: Luke Simon Olivera Yuhico, SUNY Upstate Medical University, 142 Beresford Lane, Minoa, NY 13116, USA. Email: luke_yuhico@yahoo.com
}

doi: $10.4021 / \mathrm{jmc} 617 \mathrm{w}$ the medication is critical to successful outcomes. If managed appropriately, cardiac dysfunction has been shown to resolve completely to previous baseline function. We present a case of mesalamine-induced myo-pericarditis with significant cardiac involvement and its subsequent return to normal capacity with quick recognition and prompt management.

\section{Case Report}

A 33-year-old man with history of Crohn's disease presented with a 3-day history of sharp, substernal and posterolateral chest discomfort, radiating to the back, and worsened with movement and inspiration. This was accompanied by headache, nausea, dizziness and fatigue. He denied any leg swelling, orthopnea, paroxysmal nocturnal dyspnea, cough or gastrointestinal symptoms other than constipation and mild abdominal pain, typical of his Crohn's disease. He recently underwent a routine colonoscopy, revealing a terminal ileal ulcer and scattered erythematous mucosa on the entire colon. He was started on mesalamine $800 \mathrm{mg}$ PO TID, a week prior to admisson. He had no known family history of heart disease, except for a grandfather who had a myocardial infarction in his 50's. He smoked three cigarettes per day for 10 years, but quit 3 months prior. He drank alcohol socially, about 1 - 2 exchanges per week, but denied history of alcohol or drug abuse. He denied any recent travel, sick contacts or tick bites.

On admission, he was flushed, ill-appearing, tachycardic and febrile at $39.7^{\circ} \mathrm{C}$. Mental status was normal. Exam was notable for neck rigidity but was otherwise unremarkable. No murmurs, gallops or rubs were heard. Diagnostic evaluation revealed: white blood cell count of 11,600, Westergren sedimentation rate of 92, C-reactive protein (CRP) of 172.8 and negative serial troponins. Electrocardiogram (EKG) showed sinus tachycardia. Echocardiogram showed pericardial thickening and/or small effusion but was otherwise unremarkable. Chest X-ray was negative. CT head and lumbar puncture done due to neck findings were unremarkable. A preliminary diagnosis of pericarditis was made. Workup for possible etiology was performed, including blood cultures, CSF culture and testing for influenza A $\mathrm{H} 1$ and $\mathrm{H} 3$ viruses, 


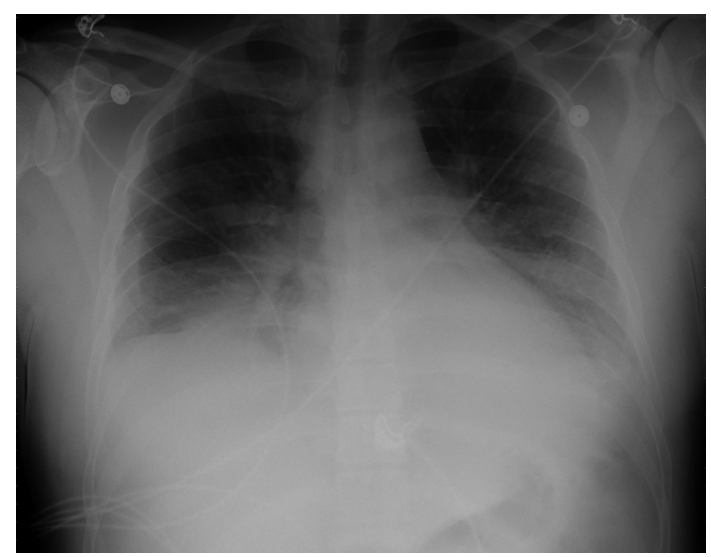

Figure 1. Chest $x$-ray showing bilateral pleural effusions as evidence of progression into congestive heart failure.

influenza B virus, respiratory syncytial virus, human metapneumovirus, parainfluenza virus types 1, 2 and 3, adenovirus and Rhinovirus/Enterovirus; all of which were negative. Mesalamine was held in view of its rare association with the disease. He was started on empiric treatment with ibuprofen.

On day 2 , he remained febrile and tachycardic. He developed increased dyspnea and, hypoxemia with p02 in the 60 's. Examination now showed jugular vein distention, s3 gallop and splinting due to marked chest pain. CT angiogram showed a tiny possible subsegmental embolus in the left lower lobe of questionable clinical significance. EKG revealed sinus tachycardia and new mild ST elevation in the lateral leads. Cardiac enzymes were negative. This evidence of new-onset cardiac dysfunction signified myocardial involvement and progression into myo-pericarditis and congestive heart failure, secondary to recent initiation of mesalamine, particularly given the absence of any alternate etiology on workup. Diuretic therapy for congestive heart failure was initiated.

On day 4, EKG revealed new non-specific intra-ventricular conduction delay, non-specific ST-T wave abnormalities, sinus tachycardia and prolonged QTc. Echocardiogram revealed new reduced left ventricular systolic function, borderline dilatation of the left atrium, trace mitral regurgitation, trace tricuspid regurgitation and a trace pericardial effusion. Chest x-ray revealed bilateral pleural effusions, confirming progression of cardiac dysfunction (Fig. 1). In addition to diuresis and ibuprofen, colchicine was then added to his regimen. Improvement was subsequently noted over the next 24 hours. Diuresis was discontinued. He was subsequently discharged on day 7 on low-dose lisinopril, metoprolol and colchicine.

On 2-week follow-up, he was completely asymptomatic. CRP was normal. EKG revealed normal sinus rhythm with normal atrioventricular and intraventricular conduction times, normal axis and no significant ST-T wave changes. Echocardiogram was within normal limits.

\section{Discussion}

Mesalamine, a 5-ASA (5-amino-2-hydroxybenzoic acid) derivative, is an active component of sulfasalazine, used to treat ulcerative colitis and maintain remission in crohn's disease [1]. Unlike sulfasalazine, mesalamine does not contain a sulfapyridine group, which is associated with side effects, such as headache, nausea, bone marrow suppression, anaphylaxis, serum sickness-like reactions and pancreatitis [2]. Its specific mechanism of action is unknown, but it is postulated to modulate local inflammatory mediators, particularly leukotrienes, and to be a free radical scavenger.

The mechanism by which it leads to myo-pericardial inflammation also remains unclear. Review of case reports showed that adverse findings of this drug were not dosedependent, ranging from $750 \mathrm{mg}$ to 4grams daily, suggesting idiosyncratic etiology [2]. In myocarditis, assumption has been that drug hypersensitivity triggers the dysfunction, rather than direct cytotoxic damage [3]. Endomyocardial biopsy done in affected tissue has documented eosinophilic infiltrates, supporting this hypothesis [4]. Discontinuation of the drug has also led to resolution of symptoms and return to normal cardiac function, as was in our case [4-6]. Similarly, inadvertent re-administration of mesalamine has led to recurrence of findings [3].

To date, only minimal data on this condition exists, despite its significant clinical impact on the population with inflammatory bowel disease (IBD). Merceron et al. described a case of myocarditis with chest pain and troponin I elevation, which improved on discontinuation of the drug, and recurred after re-administration. Although in this case, symptoms occurred years after treatment initiation, they typically occur during the first weeks of therapy, as in our case, and can lead to severe cardiac effects [3]. Other rare complications are hypersensitivity reactions, hypersensitivity pneumonitis, angioedema, pericarditis, erythroderma, toxic epidermal necrolysis, palmar-plantar erythrodysesthesia, skin rashes, and hypereosinophilia [6].

One confounder is that infrequently, IBD itself may have extraintestinal manifestations in the form of cardiac disease, most commonly pericarditis $(70 \%)$. Other manifestations would be myocarditis (10\%), myopericarditis, pericardial and pleural effusion, conduction defects, and cardiac tamponade. This can make differentiating IBD extraintestinal cardiac manifestations from a drug effect difficult. However, in the all of the cases identified as being the result of treatment toxicity, there was clear improvement after discontinuation of the drug with return to previous cardiac function, this being the likely clue toward drug reaction. For example, Garcia-Moran et al. described a severe mitral insufficiency which corrected after discontinuation of mesalazine. If resolution of symptoms is noted, then the offending drug should be contraindicated [5].

Although most case reports cite more immediate resolu- 
tion of symptoms after discontinuation of mesalamine, drug effects in our case demonstrated worsening symptoms for several days after discontinuation, and required combined therapy with both ibuprofen and colchicine prior to displaying significant improvement.

\section{Statement of Authorship}

All authors have collectively contributed to the data acquisition and review, preparation of the article, revision of the manuscript and approval for submission of this case report.

\section{Conflicts of Interest and Source of Funding}

None declared.

\section{References}

1. Podolsky DK. Inflammatory bowel disease. The New
England journal of medicine. 2002;347(6):417-429.

2. Harris A, Eswaran S, Bosworth B, Gambarin-Gelwan M, Scherl EJ. Mesalamine-induced Pneumonitis and Serum Sickness-like Reaction. Gastroenterology \& hepatology. 2007;3(11):875-877.

3. Merceron O, Bailly C, Khalil A, Pontnau F, Hammoudi N, Dorent R, Michel PL. Mesalamine-induced myocarditis. Cardiology research and practice. 2010;2010.

4. Garcia-Moran S, Saez-Royuela F, Perez-Alvarez JC, Gento E, Tellez J. Myopericarditis and mitral insufficiency associated with ulcerative colitis treated with mesalazine. Inflammatory bowel diseases. 2006;12(4):334335.

5. Stelts S, Taylor MH, Nappi J, Van Bakel AB. Mesalamine-associated hypersensitivity myocarditis in ulcerative colitis. The Annals of pharmacotherapy. 2008;42(6):904-905.

6. Kounis GN, Kouni SA, Chiladakis JA, Kounis NG. Comment: Mesalamine-associated hypersensitivity myocarditis in ulcerative colitis and the Kounis syndrome. The Annals of pharmacotherapy. 2009;43(2):393-394. 